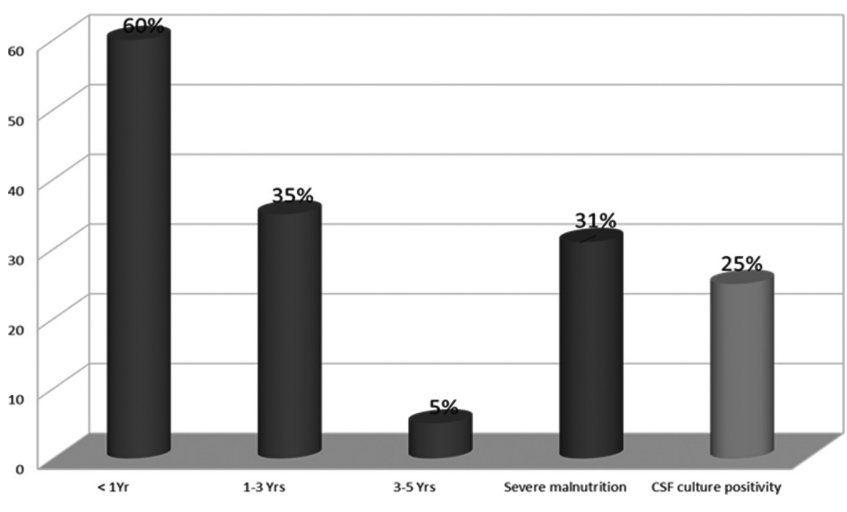

Abstract G63(P) Figure 3 Deaths in correlation with age, severe malnutrition and CSF culture positivity $n=20$

$25 \%$ with fits

$10 \%$ with crying

$23 \%$ with altered conscious level

$13 \%$ with signs of mniengeal irritation

Vaccination was incomplete in $7 \%$ unvaccinated $45 \%$ and vaccinated $48 \%$.

Complications $1 \%$ had hydrcephalus, $0.5 \%$ had subdural effusions, $4.5 \%$ had motor loss $10 \%$ have seizures maximum no of deaths less than 1 year. Malnutrition was associated with deaths in $31 \%$ and csf culture positivity in 25\% (Figures 1, 2, 3).

Conclusion Maxium no of deaths in males under 1 year, 33\% with malnutritiion, $25 \%$ with csf culture positivitity coagulase negative staph aureus and streptoccoccus pneumnia were two most commoon pathogens in culture.

Mortality under one year was maximun and associated with streptococcus pneumonia.

\section{G64(P) FOCAL SEIZURE, HEMIPARASIS, HEMIPLAGIA AND NMDA RECEPTOR ANTIBODY ENCEPHALITIS - ATYPICAL PRESENTATION}

D Onyekwere, G Margabanthu. Paediatrics, Kettering General Hospital, Kettering, UK

\subsection{6/archdischild-2015-308599.63}

Aim We present an atypical case of NMDAR Ab Encephalitis (N-methyl-D-Aspartate Receptor Antibody Encephalitis) with recurrent right sided focal seizures, right hemiparesis and hemiplegia. The youngest case in UK to be described without significant psychiatric component.

Methods 4 years 10 month old girl born in UK to Zimbabwean Parents. She had been well with not relevant family history. She presented with right sided focal seizure and reduced movement. She had 2 episodes of generalised tonic convulsions involving the right side associated with loss of consciousness which lasted for $2 \mathrm{~min}$ and then $10 \mathrm{~min} ; 5 \mathrm{~min}$ apart. The seizures was managed with diazepam. No preceding trauma, fever, neck pain, headache or vomiting. Her right sided weakness which improved within $2 \mathrm{~h}$.

She was then re admitted 4 days later with right focal seizure with persisting right sided weakness, dysarthria and right sided facial palsy. She had amnesia without any new psychotic features

Baseline blood and CSF Investigation were normal. Lyme serology, ASOT, Lupus screen, all viral PCR and throat swab were negative. ECG, Echo and EEG was normal. CSF amino acid, ammonia, Lyme titre, oligoclonal bands, mitochondrial
DNA, acylcarnitine profile was normal. MRI showed possible left MCA infarct with a picture of encephalitis with oedema.

She was treated with antibiotics, lorazepam, Aspirin and Omeprazole. Repeat MRI ruled out a stroke so Aspirin was stopped and started on IV methylprednisolone.

Repeated MRI brain suggested an inflammatory process with evidence of maturing damage suggestive of neurometabolic or neuroinflammatory disorder. Importantly the CSF and blood NMDAR antibody returned positive with rising titres of blood NMDAR ab.

Results NMDA Receptor Antibody Encephalitis was diagnosed and treated with Prednisolone $30 \mathrm{mg}$ OD for 8/52, advised avoiding live vaccines and IVIG $1 \mathrm{~g} / \mathrm{kg}$ for $2 / 7$. MMF $200 \mathrm{mg}$ BD planned for 18-24 months- dose to be adjusted based on response. Monthly NMDAR ab blood and CSF have been positive for 10 months after diagnosis. She is on Annual surveillance for tumours with MRI or USS. She continues to have physiotherapy and Neuropsychology input.

Conclusion Atypical focal seizures may need further evaluation and NMDAR ab considered with atypical presentations resulting in better outcome.

\section{G65(P) NEUROPSYCHIATRIC PRESENTATION OF ANTIBODY NEGATIVE LIMBIC ENCEPHALITIS}

K Moodley, A Menounou, A Kirby, A Durso, D Krishnakumar. Paediatrics and Child Health, Colchester General Hospital, Colchester, UK

\subsection{6/archdischild-2015-308599.64}

Autoimmune encephalitis is an increasingly recognised cause for a combination of symptoms of seizures, disturbance of memory, behaviour and cognition. Other manifestations include acute onset movement disorders, unexplained encephalopathy and refractory status epilepticus. N-methyl aspartate (NMDA) encephalitis and voltage gated potassium channel mediated (VGKC) limbic encephalitis are the two commonest causes of autoimmune encephalitis. These children initially present to general paediatricians therefore it is vital to consider this in the differential diagnosis of infective encephalitis as prompt recognition, investigation and immune therapy determines longterm outcome. Early recognition and treatment can potentially halt temporal lobe atrophy and improve outcome.

We report a 14 year old girl who presented with symptomatic, intermittent memory loss, behaviour disturbance, fatigue and cognitive change with limbic encephalitis. Initial investigations revealed negative PCR for HSV, EBV. Brain imaging detected mesial right temporal high signal area in FLAIR sequences. EEG showed epileptic inter-ictal focal abnormality over right frontal mesial temporal region. NMDA, VGKC, Anti GAD, Hu, Ma, CV2, CRMP5 and thyroid antibodies were negative. Screening for tumour with baseline abdominal ultrasound and CXR were negative. Baseline WISC testing prior to starting medications revealed her immediate memory for verbal information was in the low average range (verbal immediate index score:82,12th percentile) and her time delayed recall verbal information was in the impaired range (verbal delayed index score:72, 3rd percentile). She was treated with iv methylprednisolone followed by tapering dose of oral prednisolone. Repeat WISC assessment is due in 6 months.

Limbic encephalitis should be considered in children with prominent neuropsychiatric manifestation of encephalitis. Her future MRI reports will determine the 3 month and 6 month 
outcome for this teenager. Longterm surveillance for tumour detection is recommended in autoimmune encephalitis.

\section{G66(P) INTERESTING CASES OF PYRIDOXINE DEPENDENT EPILEPSY IN LATE CHILDHOOD}

S Jain, E Wassmer. Neurology, Birmingham Children's Hospital, Birmingham, UK

\subsection{6/archdischild-2015-308599.65}

We report cases of two children with intractable epilepsy who responded to treatment with pyridoxine in late childhood.

Case details Case 1: A 14 year old girl born following an uneventful pregnancy to non consanguinous parents. She has global developmental delay. She developed epilepsy at around 2 years of age. Her extensive investigations including an MRI of brain were normal. She had various types of seizures and these were refractory to antiepileptic medications. A trial of pyridoxine at around 13 years of age surprisingly had a very good effect on her seizure control. We are currently awaiting the result of urinary L- $\alpha$-aminoadipic semialdehyde (AASA).

Case 2: A 4 year old girl born following normal pregnancy developed infantile spasms at 4 months of age. She was treated with numerous courses of steroids with suboptimal response. Extended investigations including the MRI brain were normal. She eventually developed refractory epilepsy with cognitive decline. A trial of pyridoxine at 3 years of age achieved good control of epilepsy and improved her cognition as well. The urinary AASA was negative and the result of PNPO gene mutation is awaited.

Discussion Pyridoxine dependent epilepsy is a syndrome that usually presents with neonatal intractable seizures. It may present later in infancy or early childhood. A variety of seizure types are reported, the most common being generalised tonic clonic seizures which progress to status epilepticus. Urinary AASA is usually elevated and the genetic testing is available for this condition now. Our cases responded to the empirical trial of Pyridoxine at a much later age.

Conclusion We recommend testing for pyridoxine deficiency and therapeutic trial with pyridoxine in children with refractory epilepsy in all the age groups. Early detection and treatment of this condition not only achieves good seizure control but can possibly improve developmental outcome as well.

\section{G67(P) CHILDHOOD PARKINSONISM: A RARE COMPLICATION OF HYPOXIC BRAIN INJURY}

${ }^{1} \mathrm{M}$ Ramphul, 'A Joshi, ' $\mathrm{A}$ Maw. 'Paediatrics, Luton and Dunstable University Hospital, Luton, UK; ${ }^{2}$ Paediatric Neurology, Cambridge University Hospital, Cambridge, UK

\subsection{6/archdischild-2015-308599.66}

Case report Child A is a 13-year old boy with a history of poor adherence to his asthma treatment.

He was admitted to hospital with an acute severe exacerbation of asthma, which needed escalation of treatment to intravenous bronchodilators. Despite intensive treatment, he went on to have a cardio-respiratory arrest, necessitating five minutes of cardiopulmonary resuscitation and adrenaline prior to return of his circulation.

Child A spent a week on PICU, after which he underwent neurological rehabilitation on the ward, involving a multidisciplinary team. MRI of his brain confirmed severe hypoxic brain

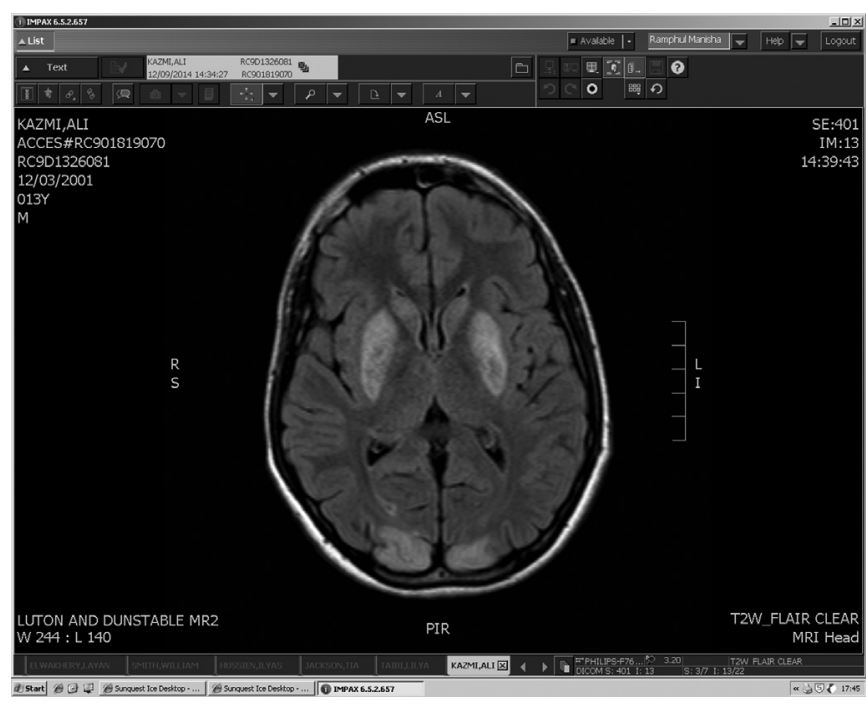

Abstract G67(P) Figure 1

injury with infarction within the basal ganglia and occipital lobe (Figure 1). Similar to other children with acquired brain injury, he demonstrated 4-limb spasticity, as well as dystonic posturing.

In addition to this, he also displayed Parkinsonian features, which is a rare complication of brain injury. He had a resting tremor, bradykinesia, rigidity and a shuffling gait, with difficulty in turning around. He displayed hypomimic facies, along with a monotonous speech. The above symptoms responded well to cocareldopa.

Discussion Poor compliance with asthma medication in children remains a significant problem with major health implications. Our case sustained a severe brain injury as a result of this. Unlike in adults where Parkinsonism is common, this condition is rare in children and easy to miss. The commonest cause of Parkinsonism is the loss of dopaminergic neurones in the substantia niagra of the basal ganglia. Child A's symptoms resolved with co-careldopa which increases dopamine levels in the brain.

We have not come across a similar paediatric case during our literature review. Paediatricians need to be vigilant to identify Parkinsonian features in children with brain injury. These typical signs become apparent to the clinician who is clearly looking out for them. Making this diagnosis correctly is important so that we can prescribe a specific anti-Parkinsonian medication, rather than make the child go through repeated failed trials with incorrect drugs. There are different classes of medication for Parkinsonism and treatment choices are largely based on data from adults.

Recognising this movement disorder is hence vital to support the rehabilitation process and optimise recovery.

\section{G68(P) A RETROSPECTIVE REVIEW OF EPILEPSY RELATED ADMISSIONS TO THE PAEDIATRIC DEPARTMENT AT A DISTRICT GENERAL NHS TRUST, ENGLAND}

O Aziz, TKW Ramcharan, D Gandhi. Paediatrics, Sandwell and West Birmingham NHS Trust, West Bromwich, UK

\subsection{6/archdischild-2015-308599.67}

Aims Child and Maternal Health (ChiMat) Observatory Data indicate our institution has higher Paediatric Emergency Epilepsy admissions and length of stay, compared to other PCTs in 